\title{
Comparative genomic analysis of Polypodiaceae chloroplasts reveals fine structural features and dynamic insertion sequences
}

Shanshan Liu', Zhen Wang ${ }^{1}$, Yingjuan Su, ${ }^{1,2^{*}}$ and Ting Wang ${ }^{3^{*}}$

\begin{abstract}
Background: Comparative chloroplast genomics could shed light on the major evolutionary events that established plastomic diversity among closely related species. The Polypodiaceae family is one of the most speciesrich and underexplored groups of extant ferns. It is generally recognized that the plastomes of Polypodiaceae are highly notable in terms of their organizational stability. Hence, no research has yet been conducted on genomic structural variation in the Polypodiaceae.

Results: The complete plastome sequences of Neolepisorus fortunei, Neolepisorus ovatus, and Phymatosorus cuspidatus were determined based on next-generation sequencing. Together with published plastomes, a comparative analysis of the fine structure of Polypodiaceae plastomes was carried out. The results indicated that the plastomes of Polypodiaceae are not as conservative as previously assumed. The size of the plastomes varies greatly in the Polypodiaceae, and the large insertion fragments present in the genome could be the main factor affecting the genome length. The plastome of Selliguea yakushimensis exhibits prominent features including not only a largescale IR expansion exceeding several kb but also a unique inversion. Furthermore, gene contents, SSRs, dispersed repeats, and mutational hotspot regions were identified in the plastomes of the Polypodiaceae. Although dispersed repeats are not abundant in the plastomes of Polypodiaceae, we found that the large insertions that occur in different species are mobile and are always adjacent to repeated hotspot regions.

Conclusions: Our results reveal that the plastomes of Polypodiaceae are dynamic molecules, rather than constituting static genomes as previously thought. The dispersed repeats flanking insertion sequences contribute to the repair mechanism induced by double-strand breaks and are probably a major driver of structural evolution in the plastomes of Polypodiaceae.
\end{abstract}

Keywords: Plastome, Polypodiaceae, Insertions, Dynamic evolution

\footnotetext{
* Correspondence: suyj@mail.sysu.edu.cn; tingwang@scau.edu.cn

'School of Life Sciences, Sun Yat-sen University, Guangzhou, Guangdong,

China

${ }^{3}$ College of Life Sciences, South China Agricultural University, Guangzhou, Guangdong, China

Full list of author information is available at the end of the article
}

(c) The Author(s). 2021 Open Access This article is licensed under a Creative Commons Attribution 4.0 International License, which permits use, sharing, adaptation, distribution and reproduction in any medium or format, as long as you give appropriate credit to the original author(s) and the source, provide a link to the Creative Commons licence, and indicate if changes were made. The images or other third party material in this article are included in the article's Creative Commons licence, unless indicated otherwise in a credit line to the material. If material is not included in the article's Creative Commons licence and your intended use is not permitted by statutory regulation or exceeds the permitted use, you will need to obtain permission directly from the copyright holder. To view a copy of this licence, visit http://creativecommons.org/licenses/by/4.0/ The Creative Commons Public Domain Dedication waiver (http://creativecommons.org/publicdomain/zero/1.0/) applies to the data made available in this article, unless otherwise stated in a credit line to the data. 


\section{Background}

Chloroplasts are the plant organelles in which photosynthesis takes place. Each chloroplast contains its own genome (plastome), which usually occurs in multiple copies within the organelle [1]. In recent years, the chloroplast (cp) genome has become a preferred target for comparative genomics because of its mostly uniparental inheritance, compact size, lack of recombination, and moderate evolutionary rate compared to the two other genomes found in plant cells (nuclear and mitochondrial genomes) [2-4]. Advances in DNA sequencing technology have provided highly efficient, cost-effective sequencing platforms, and the properties of the plastome made it one of the first candidates for high-throughput sequencing and assembly. Plastomes have now been extensively used for exploring phylogenetic relationships and understanding evolutionary processes of plants [5-7].

Ferns, which are the second largest group of vascular plants, play important ecological roles and hold a pivotal phylogenetic position [8]. The sequencing of fern plastomes has greatly increased our understanding of the plastomic diversity and evolution of this lineage. The sizes of the plastomes of ferns are highly conserved, and they usually exhibit a circular structure ranging from 120 to $170 \mathrm{~kb}$ [9]. The plastomes of ferns typically consist of four parts, including a pair of large inverted repeats (IRs), a large single copy (LSC) region, and a small single copy (SSC) region. Almost all fern IRs contains a core gene set of four ribosomal RNAs (16S, 23S, 4.5S, and $5 \mathrm{~S}$ ) and several tRNA genes (trnA-UGC, trnI-GAU, trnN-GUU, and trnR-ACG). Structurally, the plastome of fern lineages has evolved in a conservative manner. Most fern plastomes are largely collinear, requiring only a few inversions and IR expansions to account for the large-scale structural rearrangements observed among major lineages. For example, the unique chloroplast genomic rearrangement of core leptosporangiate ferns (Salviniales, Cyatheales, and Polypodiales) and Schizaeales can be explained by an expansion of the IRs and "two inversions" [10], which mainly affect the orientation and gene content of the IRs [11]. The conservative nature of the plastome makes it homogeneous enough to allow comparative studies to be conducted across higher-level taxa, but it is also sufficiently divergent to capture various evolutionary events.

The Polypodiaceae family is one of the most speciesrich groups of extant ferns [12], displaying remarkable morphological and systematic diversity. Leptosporangiate ferns diversified in an angiosperm-dominated canopy during the Cenozoic radiation period, thus establishing the diversity of the Polypodiaceae [13]. Most species within Polypodiaceae are epiphytes, and this family represents one of the most diverse and abundant groups of pantropical vascular epiphytes in tropical and subtropical forests [14, 15]. The plastomes of Polypodiaceae have undergone a variety of complex genomic reconstructions over evolutionary time, making them significantly different from those of the basal ferns (Marattiales, Ophioglossales, Psilotales, and Equisetales). Few studies have analyzed the gene content and structural changes of Polypodiaceae plastomes in detail because plastome evolution in Polypodiaceae is considered relatively dormant compared with that in other lineages. Most of the relevant research has focused on phylogenetic topics. Recent studies, however, have shown that the plastomes of polypods contain not only hypervariable regions but also widespread mobile sequences [16, 17]. Unfortunately, the corresponding studies have rarely involved Polypodiaceae because of the limited available plastome data for this group. This indicates that the currently available plastome information may be insufficient to elucidate the evolutionary patterns of the fern genome, and the exploration of the structural diversity of Polypodiaceae plastomes is also far from sufficient. Comparative genomic analyses of the chloroplasts among closely related species can generate genetic markers and provide a more exhaustive understanding of the evolutionary trajectory of the genome $[18,19]$. Nevertheless, the plastome structural and sequence homogeneity among low-level taxa makes it difficult to identify various evolutionary events. Therefore, it is necessary to compare the fine structural characteristics of Polypodiaceae plastomes to increase the understanding of the diversity and dynamic evolution of fern plastomes. Upon this premise, we performed the first family-scale comparative analysis of plastome structure and content in Polypodiaceae.

We utilized a high-throughput sequencing platform to assemble three new plastomes, two from Neolepisorus and one from Phymatosorus, and used them to perform detailed comparative analysis with the set of all previously published Polypodiaceae plastomes in an effort to: 1) characterize the genomic structure and gene content of newly sequenced plastomes; 2) examine Polypodiaceae plastome variation at the fine structural level; and 3) gain insight into the plastome evolution of Polypodiaceae.

\section{Results}

\section{Genome assembly and annotation}

Illumina paired-end sequencing generated 6,760,171, 6, 821,122 , and 6,764,140 raw reads from Neolepisorus fortunei, Neolepisorus ovatus, and Phymatosorus cuspidatus, respectively (Table 1). A total of 635,763 to 1,054, 231 clean reads were mapped to the reference plastome and 11 to 16 contigs were assembled for three species, reaching over $160 \times$ coverage on average over the plastomes. The draft plastome of $N$. fortunei, $N$. ovatus, and $P$. cuspidatus had six, two, and three gaps, respectively. 
Table 1 Details of plastome sequencing and assembly

\begin{tabular}{llll}
\hline Sample & $\boldsymbol{N}$. ovatus & N. fortunei & P. cuspidatus \\
\hline Raw Reads & $6,760,171$ & $6,821,122$ & $6,764,140$ \\
Clean reads & $5,765,899$ & $5,907,868$ & $5,880,444$ \\
Raw data & $2.03 G$ & $2.05 G$ & $2.03 G$ \\
Clean data & $1.73 G$ & $1.77 G$ & $1.76 \mathrm{G}$ \\
Contig & 13 & 11 & 16 \\
Genome coverage $(\times)$ & 313 & 292 & 160 \\
Size $(b p) / G C \%$ & $151,936 / 42.3$ & $151,915 / 41.3$ & $152,161 / 41.4$ \\
\hline
\end{tabular}

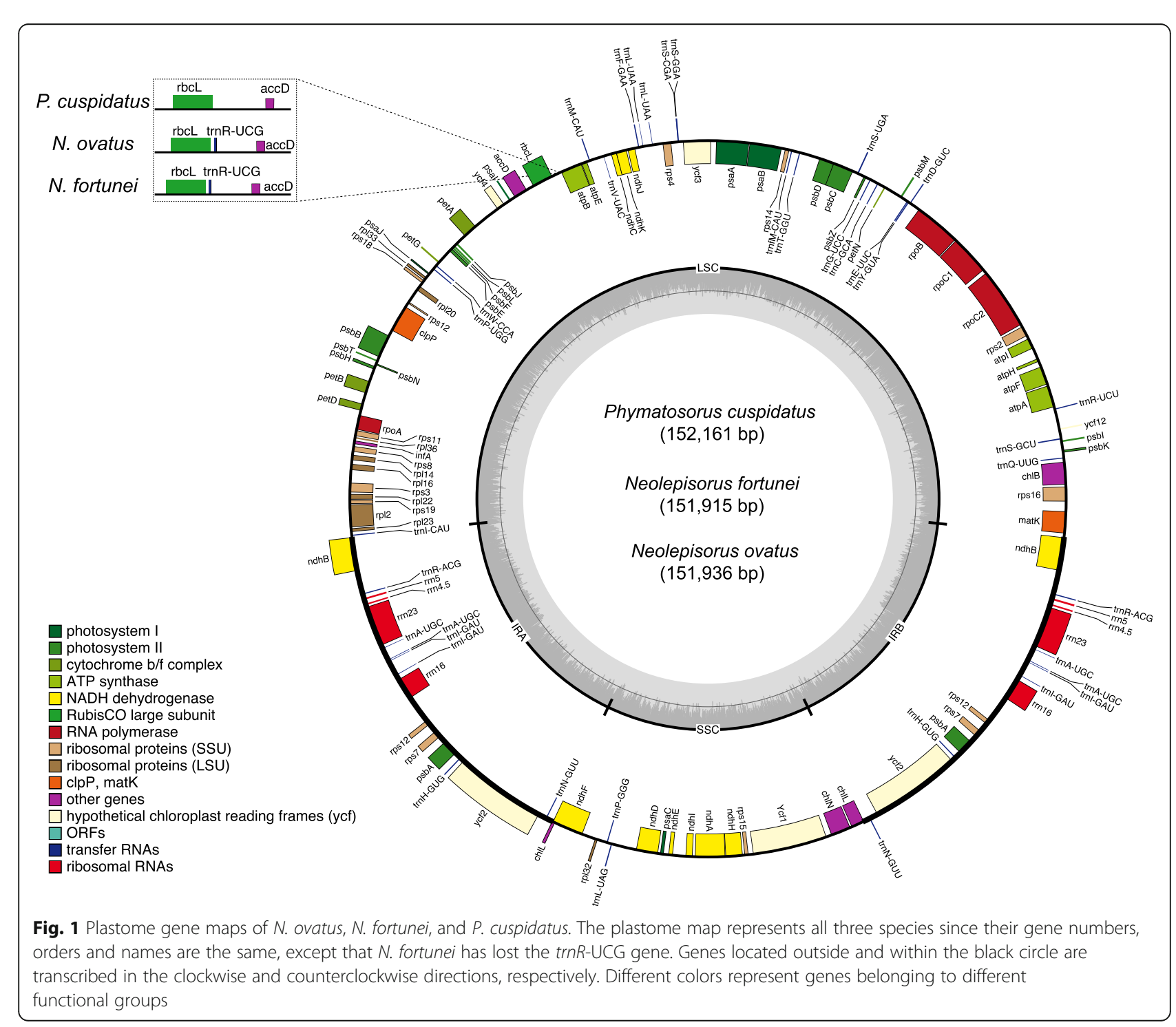

The gap regions for each resulting plastome were filled by using PCR-based sequencing with corresponding pairs of primers (Table S1). The length of the complete plastome sequences ranged from 151,915 to $152,161 \mathrm{bp}$, with an average GC content of 41.7\% (range 41.3-42.3\%; tite structure, harboring a pair of large IRs (24,609-24, $756 \mathrm{bp})$. The two IR regions divide the plastomes into an LSC region (80,670-81,175 bp) and an SSC region (21, 601-21,733 bp) (Fig. 1, Table 2). The three newly sequenced plastomes have a similar gene content, with a few notable distinctions. Compared to the other two plastomes, loss of trnR-UCG is observed in P. cuspidatus (Fig. 1). rps16 harbors an approximately 470-bp intronic deletion in $N$. fortunei (Fig. S1). Furthermore, $N$. fortunei has extra complete copies of $n d h B$ in IRb because of its IRa/LSC border adjacent to $n d h \mathrm{~B}$, whereas $N$. ovatus and $P$. cuspidatus only contains a second fragment of Table 1). All plastomes exhibited the typical quadripar- 
Table 2 Comparison of general features of the plastomes of Polypodiaceae

\begin{tabular}{|c|c|c|c|c|c|c|c|c|c|c|c|c|}
\hline \multirow{2}{*}{$\begin{array}{l}\text { Subfamily } \\
\text { Species }\end{array}$} & \multicolumn{8}{|c|}{ Microsoroideae } & \multicolumn{2}{|c|}{ Platycerioideae } & \multicolumn{2}{|c|}{ Drynarioideae } \\
\hline & NO & $N F$ & $P C$ & $P N$ & $L C$ & LHem & $L M$ & LHed & $P B i$ & $P B O$ & $D R$ & SY \\
\hline LSC (bp) & 81,117 & 80,670 & 81,175 & 81,506 & 81,093 & 81,403 & 81,244 & 81,395 & 79,002 & 82,479 & 86,040 & 80,975 \\
\hline SSC (bp) & 21,601 & 21,733 & 21,688 & 21,759 & 21,679 & 25,492 & 21,797 & 21,756 & 21,485 & 21,723 & 24,433 & 19,848 \\
\hline IR (bp) & 24,609 & 24,756 & 24,649 & 24,643 & 27,113 & 24,594 & 27,494 & 24,593 & 28,249 & 26,986 & 23,416 & 32,017 \\
\hline Size (bp) & 151,936 & 151,915 & 152,161 & 152,551 & 156,998 & 156,083 & 158,029 & 152,337 & 156,985 & 158,174 & 154,305 & 164,857 \\
\hline PGGs & 88 & 89 & 88 & 88 & 88 & 87 & 88 & 88 & 88 & 88 & 88 & 91 \\
\hline tRNAs & 35 & 35 & 34 & 35 & 35 & 34 & 35 & 35 & 33 & 35 & 35 & 35 \\
\hline rRNA & 8 & 8 & 8 & 8 & 8 & 8 & 8 & 8 & 8 & 8 & 8 & 8 \\
\hline Pseudo & 1 & 0 & 1 & 1 & 0 & 2 & 1 & 1 & 1 & 1 & 1 & 1 \\
\hline Genes & 132 & 132 & 131 & 132 & 131 & 131 & 132 & 132 & 130 & 132 & 132 & 135 \\
\hline LSC (GC\%) & 40.2 & 42.1 & 40.4 & 41.5 & 40.46 & 43.6 & 40.5 & 41.9 & 37.9 & 40.1 & 39.8 & 39.5 \\
\hline SSC (GC\%) & 37.5 & 38.8 & 37.6 & 39.0 & 37.9 & 43.0 & 37.9 & 39.7 & 34.4 & 37.4 & 36.6 & 39.8 \\
\hline IR (GC \%) & 44.9 & 45.1 & 44.8 & 45.3 & 45.4 & 45.9 & 45.3 & 45.2 & 44.9 & 45.6 & 45.1 & 42.7 \\
\hline GC (Total\%) & 41.3 & 42.3 & 41.4 & 42.4 & 41.8 & 44.2 & 41.8 & 42.7 & 39.9 & 41.6 & 40.9 & 40.8 \\
\hline
\end{tabular}

NO N. ovatus, NF N. fortunei, PC P. cuspidatus, PN P. niponica, LC L. clathratus, LHem L. hemionitideus, LM L. microphyllum, LHed L. hederaceum, PBi P. bifurcatum, $P B o$ P. bonii, DR D. roosii, SY S. yakushimensis, $P C G$ protein-coding gene, Pseudo pseudogene

$n d h B$ in IRb due to their IRa/LSC border lies within $n d h \mathrm{~B}$.

\section{Whole-chloroplast genome comparison among Polypodiaceae}

The three newly obtained plastomes of Polypodiaceae (N. ovatus, $N$. fortunei, and P. cuspidatus) were compared with nine previously published plastomes representing three subfamilies of Polypodiaceae, i.e., Microsoroideae, Platycerioideae, and Drynarioideae (Table 2). The Polypodiaceae plastomes appeared to be structurally similar to each other, showing a typical quadripartite structure consisting of two IRs separated by LSC and SSC. Overall, the analysis showed that the size of the plastome varies widely among Polypodiaceae, ranging from $151,936 \mathrm{bp}$ in $N$. ovatus to $164,857 \mathrm{bp}$ in Selliguea yakushimensis. The lengths of the LSC and SSC regions of most species are approximately $81 \mathrm{~kb}$ and $21 \mathrm{~kb}$, respectively, but these two regions of the Drynaria roosii plastome are significantly larger, reaching $86 \mathrm{~kb}$ and $24 \mathrm{~kb}$, respectively. Furthermore, the plastome of Leptochilus hemionitideus also contains a larger SSC (25,492 bp). The IR regions of the Polypodiaceae plastomes show significant variation in length compared to the SC regions, varying from 23,416 to $32,017 \mathrm{bp}$. The base composition of the Polypodiaceae plastomes is more conservative relative to their size variation. The distribution of GC content is heterogeneous, with the highest being observed in IR regions (42.1-45.9\%), followed by LSC (37.9-43.6\%), while the lowest is found in the SSC region (34.4-43.0\%) (Table 2).

The gene order of the plastomes of Polypodiaceae is almost collinear. However, the structure of the $S$. yakushimensis plastome is considerably different from the typical structure of Polypodiaceae. A notable difference in the plastome of $S$. yakushimensis is a transposition of an $\sim 6 \mathrm{~kb}$ segment spanning $n d h \mathrm{~F}$ to $c c s \mathrm{~A}$ from the SSC to the IR; this inversion appears at the junction of IRb/SSC (Fig. 2). At another junction, SSC/IRa, we observed an extensive IR expansion resulting in the duplication of $y c f 1, \operatorname{chl} l \mathrm{~L}$, and $\operatorname{chl} \mathrm{N}$ at the end of IRa. In addition, a minor inversion $(\sim 2 \mathrm{~kb})$ was detected between Lepidomicrosorum hederaceum and D. roosii plastomes, which was located in rps15-ycf1 in the SSC region of $L$. hederaceum and $r r n \mathrm{~L}-t r n \mathrm{R}$ in the LSC region of $D$. roosii, respectively (Fig. 2). Genomic structural changes that occur in intergenic regions may play an additional evolutionary role, but they are difficult to detect because intergenic regions have no coding function. This unique inversion may be related to inserted sequenced or is an intermediate form of the evolution of other plastomes. As the number of published Polypodiaceae plastomes increased, the evolutionary processes of Polypodiaceae should become clearer.

The size variations of the plastome may be a result of the dynamic changes of IR boundaries [20]. However, the Polypodiaceae plastomes exhibit high similarity at SC/IR boundaries, except in S. yakushimensis (Fig. 3). The trnN-GUU gene is located in the IR adjacent to either $n d h F$ or chlL at the SSC/IR border. The $n d h F$ and chlL genes cross the SSC/IR border, extending from 14 to $53 \mathrm{bp}$ and $51-99 \mathrm{bp}$ in the IR, respectively. The IRa/ LSC border is located within the coding region of $n d h \mathrm{~B}$ and generates a pseudogene of $1,134-2,345 \mathrm{bp}$ at the $\mathrm{LSC} / \mathrm{IRb}$ border in 11 of the plastomes, except for that of $N$. fortunei. The LSC/IRb border is located within or 


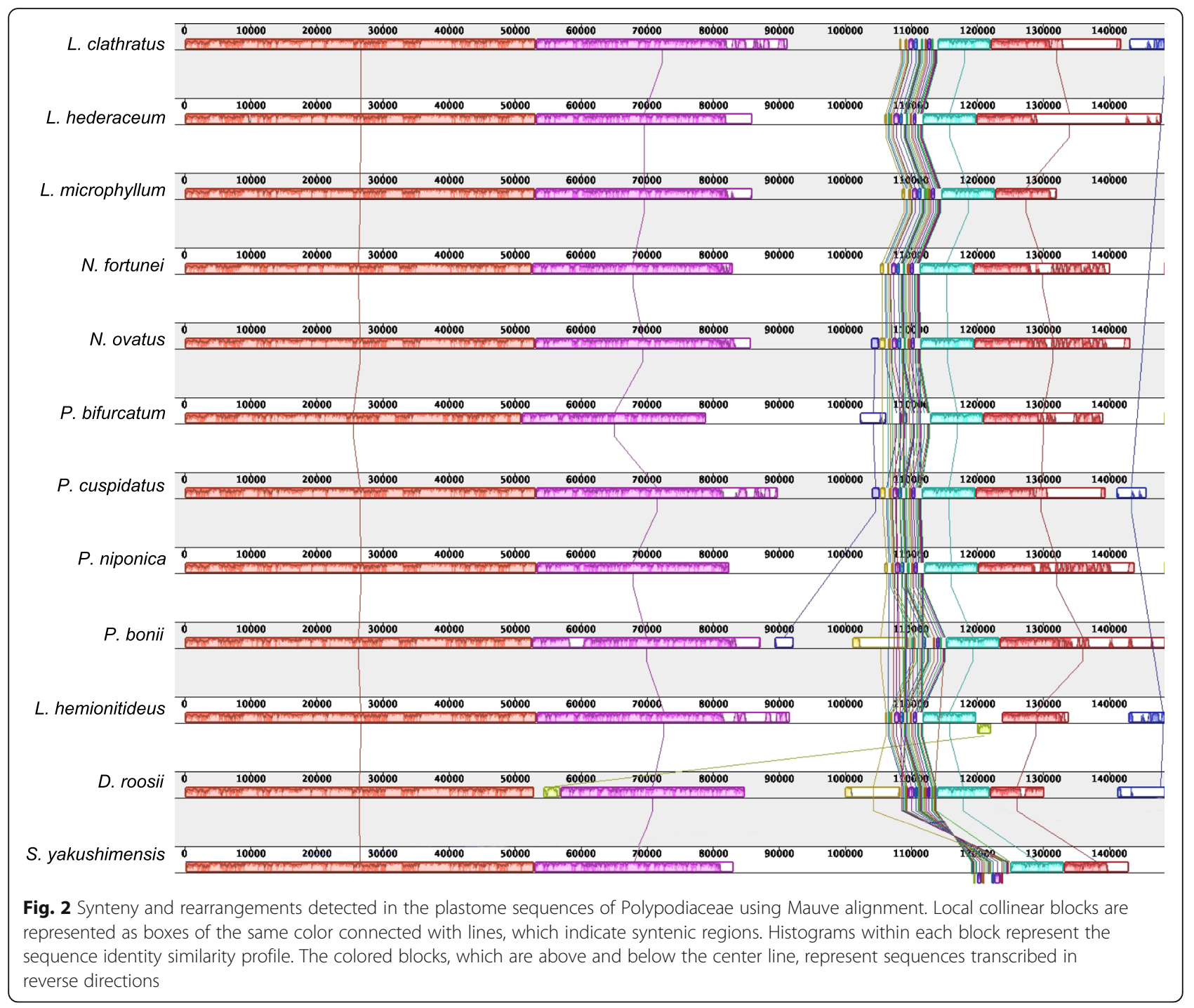

next to the trnI-CAU gene. In contrast to the subtle changes in IR boundaries, the IR of $S$. yakushimensis has experienced extensive expansion, capturing the $y c f 1$, chlL, and $\operatorname{chl} \mathrm{N}$ genes in the SSC region. This expansion, combined with the inversion of the $n d h \mathrm{~F}$-ccsA region, causes a unique SSC/IR boundary in the S. yakushimensis plastome (Fig. 3). The SC/IR boundaries of the Polypodiaceae plastomes are highly similar but not identical, indicating that the expansion and contraction of IR is an independent and recurrent phenomenon in evolution. Furthermore, the change in IR boundaries is not sufficient to cause a large difference in genome size, and we believe that microstructural changes (such as insertions and deletions) may be responsible for the difference.

There are some variations in the gene content of Polypodiaceae plastomes due to varying degrees of IR boundary changes and inversions. trnR-UCG exists in all Polypodiaceae species except for $L$. hemionitideus, $P$. cuspidatus, and Platycerium bifurcatum. trnV-UAC is also absent in the plastome of $P$. bifurcatum. Another difference is that the plastome of $N$. fortunei contains an additional intact $n d h B$ gene in the IR region, whereas only one $n d h B$ fragment exists in other species. Furthermore, close inspection of the gene annotations of the 12 Polypodiaceae plastomes indicated that the rpoC1 gene of the L. hemionitideus has been pseudogenized by a frameshift mutation.

\section{Sequence diversity and mutational hotspots of the Polypodiaceae plastome}

The multiple sequence alignments performed in mVISTA software showed the similarity of the whole sequences of the plastomes of the 12 Polypodiaceae species analyzed (Fig. 4). Lower divergence was found in the IR and protein-coding regions than in the single-copy and noncoding regions. Nevertheless, we found that obvious large inserted fragments were present in the rrn16-rps 12 spacer of the IRs in the plastomes of Lepisorus clathratus, 


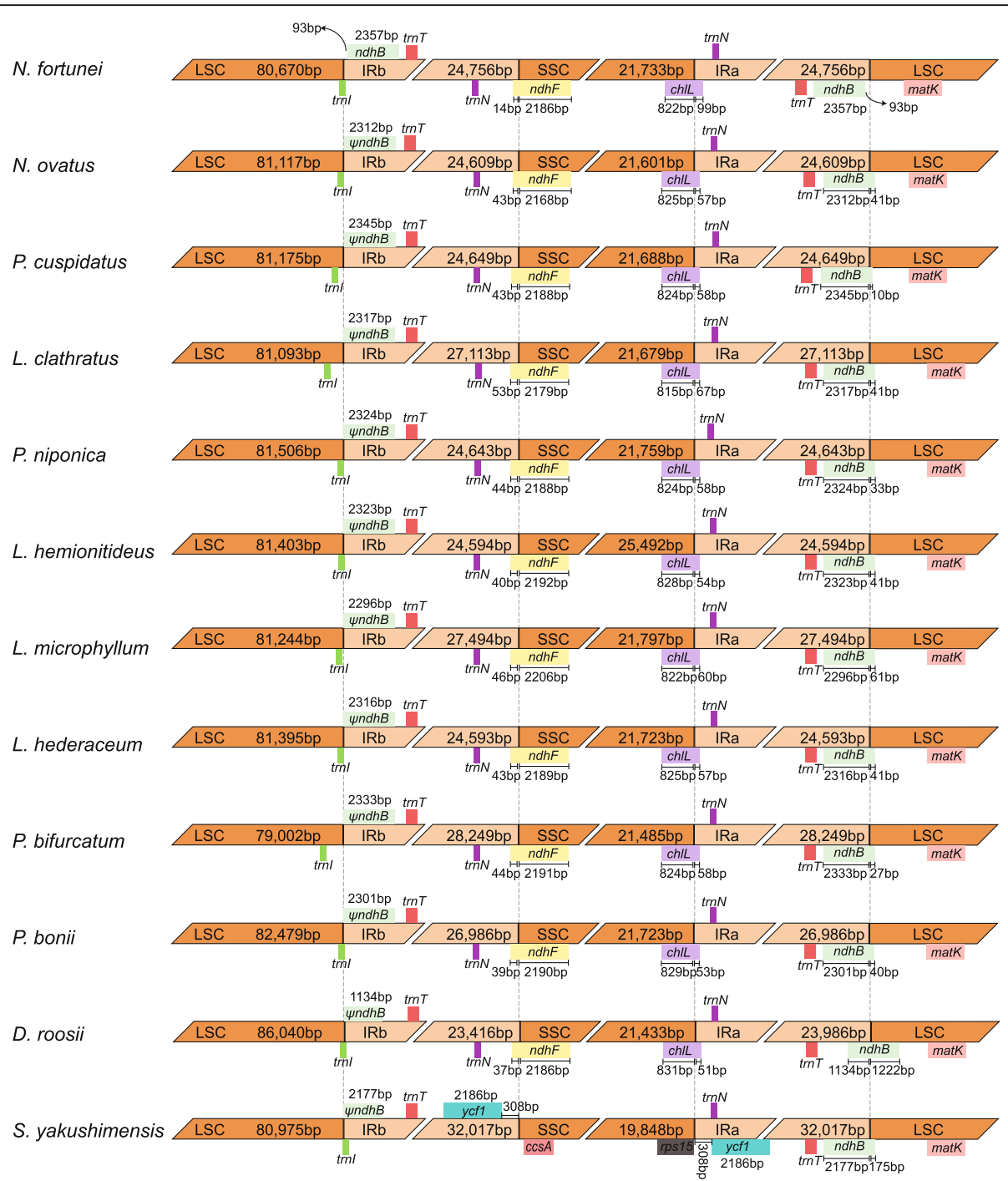

Fig. 3 Comparison of the border positions of LSC, SSC, and IR regions among the plastomes of Polypodiaceae species. $\psi$ refers to the pseudogene of $n d h B$ at the LCS/IRb border

Pyrrosia bonii, and P. bifurcatum. To detect mutational hotspots in the plastomes of Polypodiaceae, slidingwindow analysis was performed on the whole-genome alignments of the sequences using DnaSP v5.0. The results showed that the sequence variation between the plastomes of Polypodiaceae was relatively low, with nucleotide diversity $(\mathrm{Pi})$ ranging from 0.00232 to 0.20028 . Overall, the SSC region exhibited the highest sequence variation, with an average Pi of 0.10103 , followed by the LSC and IR regions, with average $\mathrm{Pi}$ values of 0.07317 and 0.02676 , respectively. A total of nine highly divergent loci were identified in the plastomes of Polypodiaceae, including matK-rps 16 , rps16, trnC-trnG, psbZ-psbC, psbD-trnT, trn $\mathrm{P}-p s a \mathrm{~J}$, and $r p l 2-t r n I$, located in LSC; rrn16-rps12, located in the IR region; and one protein-coding gene, $y c f 1$, located in SSC. The $n d h \mathrm{~F}-c c s \mathrm{~A}$ region in SSC showed a higher Pi value than the other loci, most likely due to the inversion occurring in this region, which resulted in higher sequence variation (Fig. S2). Therefore, it may not be categorized as a common mutational hotspot in the Polypodiaceae.

\section{Analysis of SSRs and repeat sequence in Polypodiaceae plastomes}

Simple sequence repeats (SSRs), or microsatellites are short, tandemly repeated DNA motifs of 1-6 nucleotides [21]. They can exhibit high polymorphism and mutation rates, which contribute to estimates of genetic variation [22]. In this study, very similar numbers of potential cpSSRs were identified from the plastomes of the 12 Polypodiaceae species by using MISA. The total number of SSRs in Polypodiaceae ranged from 38 to 51. Four kinds of SSRs were detected: mononucleotides, 


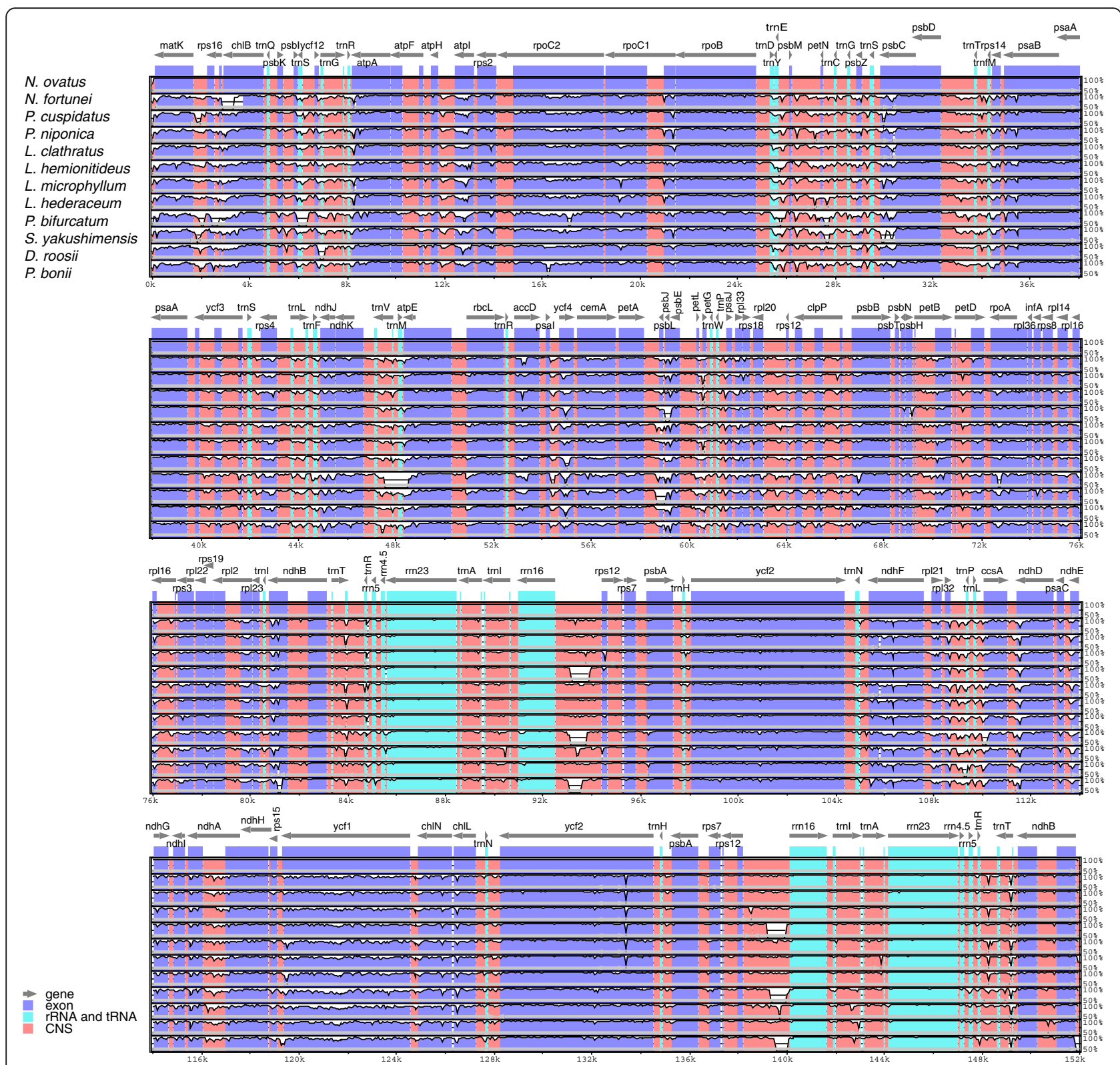

Fig. 4 Visualization of the alignment of the Polypodiaceae plastome sequences, with N. fortunei as the reference. Gray arrows above the alignment indicate genes, including their orientation and position. The vertical scale represents the percentage of identity, ranging from 50 to 100\%. Genome regions are color-coded as protein-coding genes, tRNAs, rRNAs and conserved noncoding sequences (CNSs)

dinucleotides, trinucleotides, and tetranucleotides (Fig. 5). However, tetranucleotide repeats were discovered in only the plastomes of $L$. clathratus, $L$. hemionitideus, $L$. hederaceum, S. yakushimensis, and D. roosii. Different SSR motifs appeared at different frequencies in these plastomes. The most abundant observed repeats were mononucleotides, accounting for approximately 62.8$88.3 \%$ of the total number of SSR loci, followed by smaller numbers of dinucleotide (8.7-20.9\%) and trinucleotide (6.6-22.5\%) repeats, whereas tetranucleotide repeats were the least common $(0-4.4 \%)$.
We found that the predominant mononucleotide repeats in all analyzed species with the exception of $D$. roosii, S. yakushimensis, and $P$. bifurcatum were G/C tandem motifs, which accounted for 53.3 to $100 \%$ of the mononucleotide repeats in the Polypodiaceae plastomes (Fig. 6). The Polypodiaceae species included in this study all exhibited similar SSR distribution patterns in the plastome. SSRs were much more frequently located in the LSC region (48.0-71.1\%) than in IR (10.5-36.0\%) and SSC regions $(9.3-22.0 \%)$. Furthermore, the majority of the identified SSRs were located in intergenic spacers, 


\section{Frequency of SSR type}

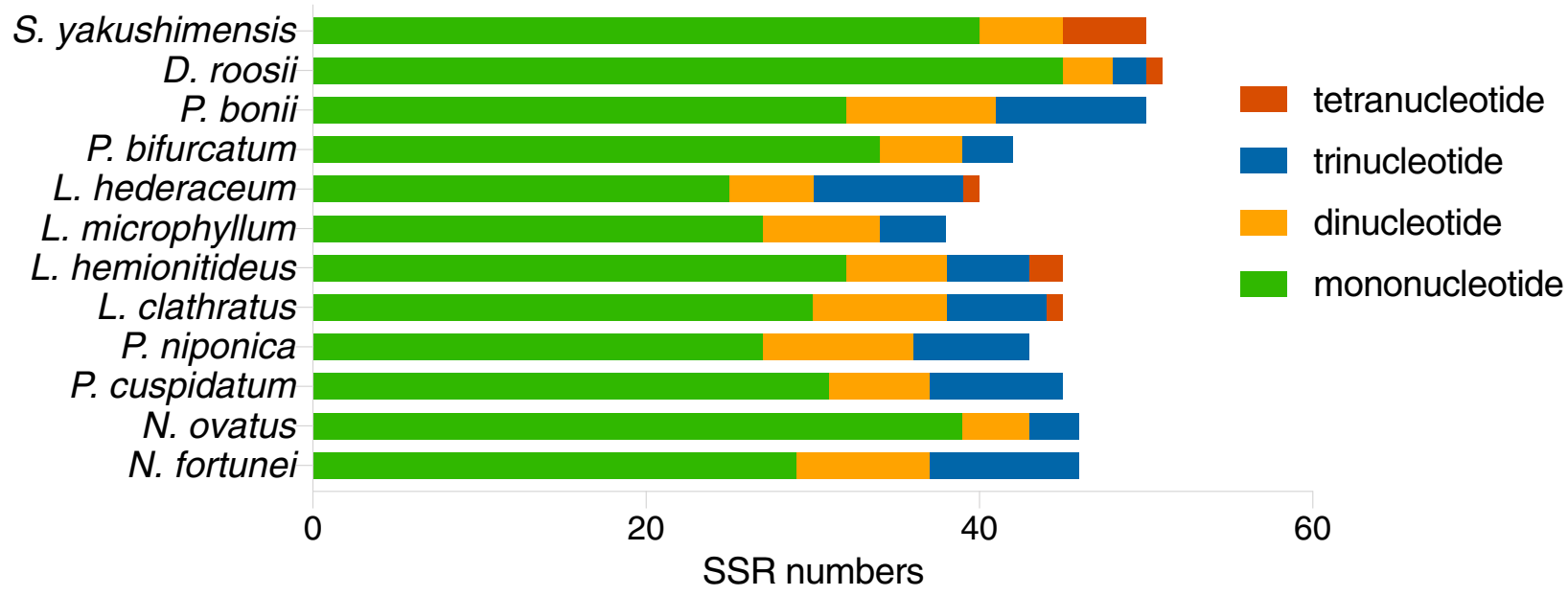

Fig. 5 Types and numbers of cpSSRs in the Polypodiaceae plastomes

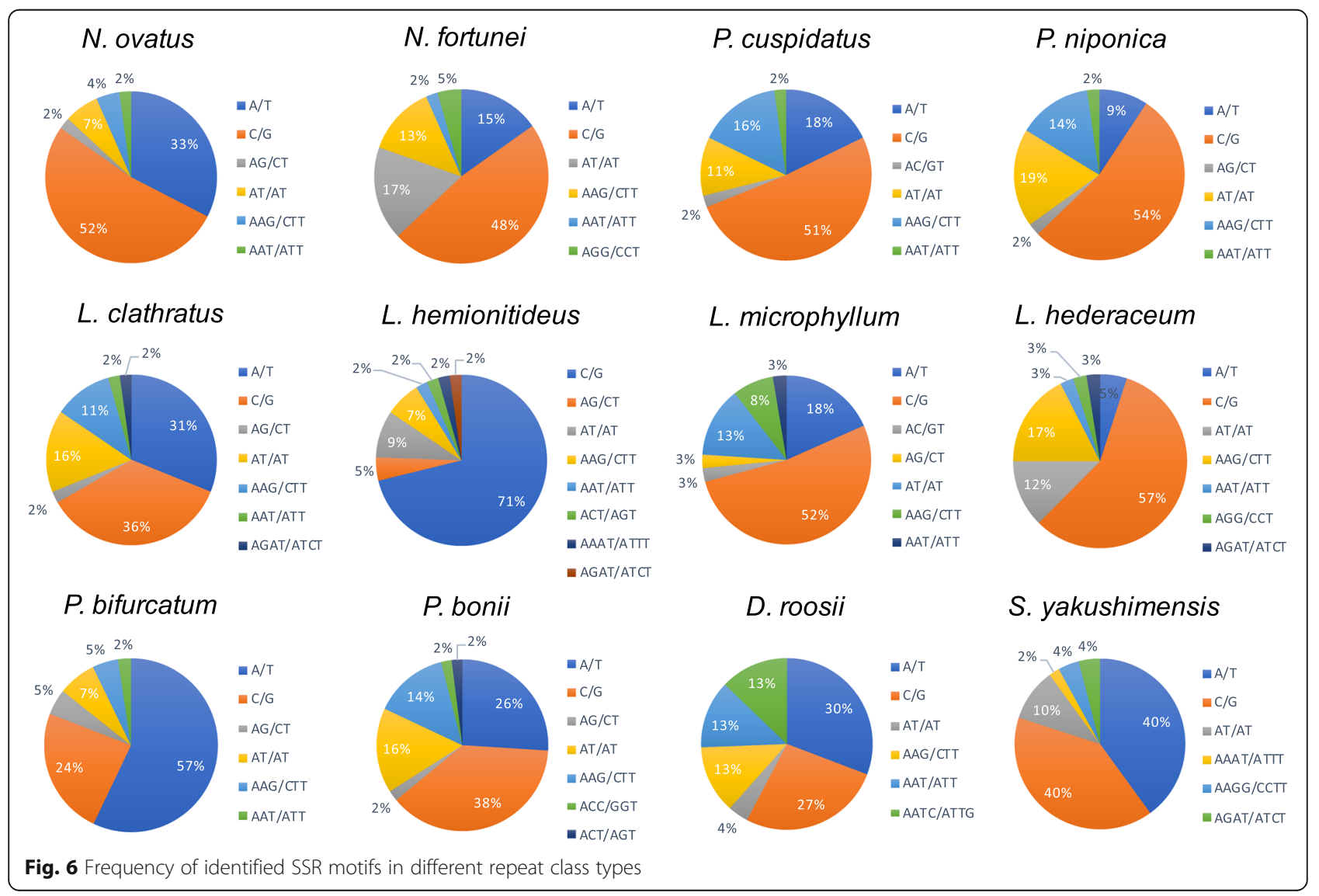


accounting for $66.7-83.3 \%$ of all SSRs detected. SSRs dispersed in intronic regions were the second most common category (9.5-28.9\%). The fewest SSRs were located in coding genes, which accounted for only $4.0-14.0 \%$ of all SSR loci (Table S2).

Forward, palindromic and reverse repeats of more than 30 bp with a sequence identity $\geq 90 \%$ were detected in the Polypodiaceae plastomes using REPuter. The results showed that the numbers of repeats in the plastomes of Polypodiaceae varied considerably, ranging from 9 in L. hemionitideus to 146 in P. bifurcatum. These long repeats ranged from 30 to $307 \mathrm{bp}$ in length and were repeated twice. Species showed some variation in the number of long repeat sequences located in intergenic spacers and coding genes. Most repeats were distributed in intergenic spacer regions, and the rest were distributed in coding genes and intronic regions (Table S3). We detected some species-specific intergenic spacers with rich repeats, including the $r b c L$-trnR-UCG intergenic spacer of the LSC region of $D$. roosii, the rps12-rrn16 intergenic spacer of the IR regions of $L$. clathratus and $P$. bifurcatum, the rps7-psbA intergenic spacer of the IR region of Lemmaphyllum microphyllum, and the $n d h \mathrm{~A}$ intronic/chlL-chlN intergenic spacer of the SC/IR junction of $P$. bonii.

Tandem Repeats Finder v4.09 [23] was further used to identify the tandem repeats present in Polypodiaceae plastomes, with the minimum identity and size of the repeats set to $90 \%$ and $15 \mathrm{bp}$ in unit length. A small number of tandem repeats were detected in all species except for P. bifurcatum and L. hemionitideus (Table 3). Among the species in which tandem repeats were detected, L. microphyllum exhibited the most repeats, and $P$. bonii exhibited the fewest. The intergenic spacers rrn16-rps12, rps7-psbA, and rbcL-trnR-UCG were regions containing abundant repeat sequences in the plastomes of L. clathratus, L. microphyllum, and $D$. roosii, respectively. All detected tandem repeats were distributed in noncoding regions, and the proportions of tandem repeats located in intergenic spacers were higher than those in intronic regions in Polypodiaceae species (Table 3).

\section{Dynamic insertion sequences in Polypodiaceae plastomes}

Through a detailed whole-genome alignment, we found that there are large insertions in some regions of Polypodiaceae plastomes, including the rrn16-rps 12 spacers of $L$. clathratus, $P$. bifurcatum, and $D$. roosii ( 2400-3500 bp insertions); the rps7-psbA spacer of L. microphyllum ( $3000 \mathrm{bp}$ insertion); the $r b c \mathrm{~L}-t r n \mathrm{R}$ spacer of $D$. roosii ( $\sim 4000$ bp insertion); the petA-psaJ spacer of $P$. bonii ( $1700 \mathrm{bp}$ insertion); and the rps 15 -ycf 1 spacer of $L$. hederaceum ( $3700 \mathrm{bp}$ insertion) (Table 4). The identity of the insertion
Table 3 Size, number, and distribution of tandem repeats in the plastomes of Polypodiaceae

\begin{tabular}{|c|c|c|c|c|}
\hline Sample & Motif & Count & Start-end & Location \\
\hline \multirow[t]{3}{*}{ N. ovatus } & 19 & 4 & $83,880-83,955$ & trnT-UGU intron \\
\hline & 23 & 2.3 & $108,602-108,647$ & rp/21-rp/32 \\
\hline & 19 & 4 & $149,099-149,174$ & $\operatorname{trnT-UGU}$ intron \\
\hline \multirow[t]{3}{*}{ N. fortunei } & 26 & 2 & $27,472-27,523$ & psbM-petN \\
\hline & 15 & 3 & $52,744-52,788$ & $\operatorname{trnR}$-UCG-accD \\
\hline & 15 & 3.9 & $116,723-116,781$ & ndhA-intron \\
\hline \multirow[t]{3}{*}{ P. cuspidatus } & 45 & 2 & 1719-1809 & matK-rps 16 \\
\hline & 26 & 2.5 & $2183-2248$ & matK-rps 16 \\
\hline & 15 & 3 & $113,805-113,849$ & psaC-ndhE \\
\hline \multirow[t]{2}{*}{ P. niponica } & 18 & 3 & $25,927-25,980$ & rpoB-trnD-GUC \\
\hline & 15 & 5 & $53,389-53,463$ & $\operatorname{trn} R-U C G-a c c D$ \\
\hline \multirow[t]{9}{*}{ L. clathratus } & 24 & 2 & 1894-1941 & matK-rps 16 \\
\hline & 22 & 2 & $21,463-21,506$ & rpoCl intron \\
\hline & 26 & 2 & $93,242-93,293$ & $\operatorname{rrn} 16-r p s 12$ \\
\hline & 27 & 3 & $93,291-93,380$ & $\operatorname{rrn} 16-r p s 12$ \\
\hline & 69 & 2 & $93,405-93,542$ & $\operatorname{rrn} 16-r p s 12$ \\
\hline & 46 & 4 & $93,470-93,653$ & rrn16-rps12 \\
\hline & 46 & 4 & $144,421-144,604$ & rps12-rrn16 \\
\hline & 27 & 3 & $144,716-144,796$ & rps12-rrn16 \\
\hline & 18 & 4 & $144,797-144,848$ & rps12-rrn16 \\
\hline \multirow[t]{10}{*}{ L. microphyllum } & 30 & 1.9 & $12,813-12,870$ & atpH-atpl \\
\hline & 18 & 3.1 & $97,069-97,123$ & rps7-psbA \\
\hline & 47 & 2.2 & $98,673-98,773$ & rps7-psbA \\
\hline & 27 & 1.9 & $98,749-98,800$ & rps7-psbA \\
\hline & 27 & 2.9 & $98,870-98,948$ & rps7-psbA \\
\hline & 15 & 5 & $119,960-120,034$ & ndhA intron \\
\hline & 27 & 2.9 & $140,326-140,404$ & psbA-rps7 \\
\hline & 27 & 1.9 & $140,474-140,525$ & $p s b A-r p s 7$ \\
\hline & 47 & 2.2 & $140,501-140,601$ & psbA-rps7 \\
\hline & 18 & 3.1 & $142,151-142,205$ & $p s b A-r p s 7$ \\
\hline \multirow[t]{2}{*}{ L. hemionitideus } & 22 & 2 & $19,153-19,197$ & $r p o C 2-r p o C 1$ \\
\hline & 25 & 1.9 & $74,525-74,572$ & rps11-rp/36 \\
\hline P. bonii & 23 & 2 & $120,079-120,124$ & $n d h A$ intron \\
\hline \multirow[t]{5}{*}{ D. roosii } & 146 & 2 & $5433-5711$ & $\operatorname{trn} Q-U G G-p s b K$ \\
\hline & 23 & 2 & $19,077-19,122$ & rpoC2-rpoC1 \\
\hline & 18 & 3 & $54,433-54,486$ & rbcL-trnR-UCG \\
\hline & 47 & 3 & $54,550-55,871$ & $r b c L-\operatorname{trn} R-U C G$ \\
\hline & 42 & 2 & $54,644-54,727$ & $r b c L-t r n R-U C G$ \\
\hline \multirow[t]{2}{*}{ S. yakushimensis } & 26 & 2 & $26,779-26,831$ & psbM-petN \\
\hline & 28 & 3.2 & $125,129-125,218$ & $n d h F-n d h D$ \\
\hline
\end{tabular}


sequences in the rrn16-rps 12 spacers of L. clathratus, $P$. bifurcatum and $D$. roosii was calculated using MegAlign v8.1.3 [24]. The pairwise alignments showed that the identity of the insertions in the three plastomes was only $48.3-50.1 \%$, indicating that these insertions may have different origins (Fig. S3).

Robison et al. [17] previously proposed the concept of MORFFO (Mobile Open Reading Frames in Fern Organelles), which are a set of mobile insertion sequences that are widely present in fern organelles. To verify whether the insertions detected in the Polypodiaceae plastomes are consistent with the MORFFO sequences, MORFFO sequences were determined by local BLAST searches $[25,26]$ using the database established from the 12 Polypodiaceae plastomes, with the consensus sequences of morffo1, morffo2, and morffo3 as queries. Furthermore, to examine whether the insertions identified in this study present mobile properties, these sequences were subjected to local BLAST searches. Our results showed that morffo 1 presents similarity to the petA-psbJ fragment of $P$. bonii (71.2\%) and the rrn16-rps12 fragment of $P$. bifurcatum (70.3\%). Morffo2 was detected in rrn16rps12 of $P$. bifurcatum and ycf1-ccsA of S. yakushimensis, with identities of $71.0 \%$ and $67.9 \%$, respectively (Table 5).

Surprisingly, the insertions contained in different species show significant BLAST hits against each other, which suggests that DNA fragments may have been transferred from one plastome to another. For example, a fragment of the L. clathratus insertion shows high sequence similarity to the rps7-psbA insertion located in the IR of L. microphyllum, rbcL-trnR located in the LSC of D. roosii, and rps15-ycf1 located in the SSC of $L$. hemionitideus. Morffo1 is located within the P. bifurcatum-P. bonii consensus insertion fragment, but morffo 2 does not show overlap with the insertions detected in this study (Table 5). Previous studies have revealed that MORFFO-like sequences are often associated with structural changes in the genome and may be the main driving force for structural evolution in the plastome. In this study, a number of movable insertions were found in the relatively static plastomes of Polypodiaceae, indicating that the different insertion fragments arising during the evolution of genome structure may have different functions.

\section{Discussion}

\section{Plastome variation across Polypodiaceae}

Our comparative analysis of 12 species from Microsoroideae, Platycerioideae, and Drynarioideae showed that the length of the plastomes varies greatly in Polypodiaceae, even within the same subfamily. Generally, dynamic expansions or contractions in IR boundaries are considered a primary mechanism leading to the size variation of land plant plastomes [20]. Although there are differences in the IR boundaries between Polypodiaceae plastomes, they also exhibit obvious similarities. Therefore, the minor shifts in the IR boundaries of the Polypodiaceae plastomes are expected to be insufficient to account for marked differences in genome size. For example, in Microsoroideae, we found that despite the IR boundaries being deeply conservative, the IR regions of $L$. clathratus and L. microphyllum are approximately $2500 \mathrm{bp}$ longer than those of other species, and the SSC region of $L$. hemionitideus is approximately $3700 \mathrm{bp}$ longer than those of other species. A fine-scale analysis of the plastome sequence data of Polypodiaceae revealed several large insertions in the specific intergenic spacers of IR or $\mathrm{SC}$ regions that correspond to the observed genome size differences. This situation corresponds well with those in the species of the other two subfamilies, with the

Table 4 Comparison of the length of intergenic regions containing large insertions in the plastomes of Polypodiaceae

\begin{tabular}{|c|c|c|c|c|c|}
\hline Species & rrn16-rps12 & rps7-psbA & rbcL-trnR & petA-psaJ & rps15-ycf1 \\
\hline N. ovatus & $1896 \mathrm{bp}$ & $478 \mathrm{bp}$ & $128 \mathrm{bp}$ & $697 \mathrm{bp}$ & $311 \mathrm{bp}$ \\
\hline N. fortunei & 1924 bp & $475 \mathrm{bp}$ & 123 bp & 682 bp & $261 \mathrm{bp}$ \\
\hline P. cuspidatus & 1902 bp & 482 bp & 130 bp & $679 \mathrm{bp}$ & $313 b p$ \\
\hline P. niponica & 1916 bp & 485 bp & $123 \mathrm{bp}$ & $681 \mathrm{bp}$ & 311 bp \\
\hline L. clathratus & $4420 \mathrm{bp}$ & 479 bp & $123 \mathrm{bp}$ & $464 \mathrm{bp}$ & 312 bp \\
\hline L. hemionitideus & $1903 \mathrm{bp}$ & $473 b p$ & $126 \mathrm{bp}$ & 631 bp & 4069 bp \\
\hline L. microphyllum & 1893 bp & 3403 bp & 127 bp & $686 \mathrm{bp}$ & 300 bp \\
\hline L. hederaceum & 1893 bp & 469 bp & $123 \mathrm{bp}$ & $693 \mathrm{bp}$ & 308 bp \\
\hline P. bifurcatum & 5476 bp & 479 bp & $125 \mathrm{bp}$ & 717 bp & 306 bp \\
\hline P. bonii & 4362 bp & 470 bp & 123 bp & 2475 bp & 304 bp \\
\hline D. roosii & 1902 bp & $475 \mathrm{bp}$ & 4107 bp & $669 \mathrm{bp}$ & 305 bp \\
\hline S. yakushimensis & 1377 bp & 364 bp & 126 bp & $677 \mathrm{bp}$ & 309 bp \\
\hline
\end{tabular}

Regions with large insertions in different species are highlighted in bold. 
Table 5 Results of matches for insertion sequences within Polypodiaceae identified using local BLAST searches

\begin{tabular}{|c|c|c|c|c|c|c|}
\hline Query & Subject & $\%$ Identity & Alignment length & Start & End & Location \\
\hline \multirow[t]{2}{*}{ morffo 1} & P. bonii & 71.2 & 1217 & 59,496 & 58,295 & petA-psbJ \\
\hline & P. bifurcatum & 70.3 & 1254 & 93,132 & 91,893 & rrn16-rps 12 \\
\hline \multirow[t]{2}{*}{ morffo2 } & P. bifurcatum & 71.0 & 1668 & 95,199 & 93,541 & rrn16-rps12 \\
\hline & S. yakushimensis & 67.9 & 1121 & 117,503 & 118,561 & $y c f 1-\operatorname{ccs} A$ \\
\hline \multirow{6}{*}{$\begin{array}{l}\text { L. clathratus } \\
\text { (rrn16-rps12) }\end{array}$} & L. microphyllum & 91.5 & 1276 & 97,718 & 96,461 & rps7-psbA \\
\hline & L.microphyllum & 88.1 & 796 & 98,773 & 97,983 & rps7-psbA \\
\hline & D. roosii & 92.0 & 647 & 54,866 & 55,512 & $r b c L-t r n R$ \\
\hline & D. roosii & 86.7 & 668 & 55,779 & 56,443 & rbcL-trnR \\
\hline & L. hemionitideus & 81.4 & 737 & 121,798 & 121,063 & rps15-ycf1 \\
\hline & L. hemionitideus & 71.3 & 523 & 120,796 & 120,281 & rps15-ycf1 \\
\hline \multirow{3}{*}{$\begin{array}{l}\text { P. bifurcatum } \\
\text { (rrn16-rps12) }\end{array}$} & P. bonii & 88.8 & 1502 & 58,225 & 59,724 & petA-psbJ \\
\hline & P. bonii & 84.7 & 698 & 59,636 & 60,323 & petA-psbJ \\
\hline & S. yakushimensis & 70.5 & 831 & 118,643 & 117,834 & $y c f 1-\operatorname{ccs} A$ \\
\hline \multirow{4}{*}{$\begin{array}{l}\text { L. microphyllum } \\
\text { (rps7-psbA) }\end{array}$} & D. roosii & 87.0 & 1527 & 56,416 & 54,895 & $r b c L-t r n R$ \\
\hline & L. clathratus & 91.5 & 1276 & 95,574 & 94,305 & rrn16-rps 12 \\
\hline & L. clathratus & 88.1 & 796 & 94,304 & 93,538 & rrn16-rps 12 \\
\hline & L. hemionitideus & 81.8 & 1425 & 120,281 & 121,697 & rps15-ycf1 \\
\hline \multirow{4}{*}{$\begin{array}{l}\text { D. roosii } \\
\text { (rbcL-trnR) }\end{array}$} & L. microphyllum & 87.0 & 1527 & 98,595 & 97,078 & rps7-psbA \\
\hline & L. hemionitideus & 79.3 & 1388 & 121,661 & 120,281 & rps15-ycf1 \\
\hline & L. clathratus & 92.0 & 647 & 93,658 & 94,304 & rrn16-rps12 \\
\hline & L. clathratus & 86.7 & 668 & 94,307 & 94,969 & rrn16-rps 12 \\
\hline P. bonii (petA-psaJ) & P. bifurcatum & 88.8 & 1502 & 91,825 & 93,310 & rrn16-rps12 \\
\hline
\end{tabular}

exception of S. yakushimensis. Therefore, in most species of Polypodiaceae, the main reason for the difference in plastome size is not the variation in SC/IR boundaries but the large fragment insertions that occur in different species. Selliguea yakushimensis of Drynarioideae exhibits the largest IRs identified in the fern lineage to date, mainly because its IR boundary has extended toward the SSC, causing the $y c f 1$, chlL, and $c h l N$ genes to be captured within the IR region. Therefore, we cannot discuss the differences in the size of the plastomes only from the perspective of the expansion and contraction of the IR boundary because the conservative nature of the plastome itself will cause researchers to ignore other factors.

It is generally recognized that plastome evolution of Polypodiaceae has mostly stabilized, and structural changes such as rearrangements occur rarely. By contrast, the results presented here indicate that the plastome of S. yakushimensis is highly unusual in some respects, containing not only a large-scale IR expansion exceeding several $\mathrm{kb}$ but also a unique inversion. The large-scale expansion of the IR presumably occurred through double reciprocal recombination between IR segments during replication [27]. Numerous dispersed repetitive sequences located at the original SSC/IR junction (chlL/trnN-GUU) were detected in the $S$. yakushimensis plastome, coinciding exactly with the mechanism of IR expansion; that is, repeat sequences provide the potential for genome rearrangement within or between molecules by homologous recombination $[27,28]$. In general, the homogeneity of plastome structural changes is low relative to the sequence data. The structural changes in the S. yakushimensis plastome can therefore serve as specific genetic markers in species discrimination or phylogenetic analyses. Unfortunately, there is no way to determine which event took place first because IR expansion has occurred at the SSC/IRa boundary, while inversion has occurred near the IRb/ SSC boundary. Consequently, it is necessary to sequence more plastomes in Polypodiaceae to improve our understanding of the evolutionary trajectory of the plastome.

Whole-plastome alignments can elucidate the level of sequence divergence and easily identify large indels, which are extremely useful for phylogenetic analyses and plant identification. In the present study, our results showed that the IRs present lower sequence divergence than the SC regions. This phenomenon is considered to be a result of copy correction between IR sequences and the elimination of deleterious mutations by gene conversion [29]. Moreover, sequence differences among the 
Polypodiaceae plastomes were evident in the intergenic spacers, suggesting greater conservation in coding regions than in noncoding regions. Nine divergence hotspots between Polypodiaceae species were identified, including matK-rps16, rps16, trnC-trnG, psbZ-psbC, psbD-trn T, trnP-psa), rpl2-trnI, rrn16-rps 12 , and $y c f 1$, among which nine loci were located in SC and one was located in an IR. Although the $n d h \mathrm{~F}$-ccsA regions also present higher Pi values, they may not be suitable as general mutational hotspots for Polypodiaceae due to the existence of inversion in this region of the S. yakushimensis plastome. Thus, we must conduct careful data exploration when screening universal mutational hotspots to avoid confusion by plastome structural changes.

In this study, we found many repeat regions, including forward repeats, palindromic repeats, and reverse and tandem structures, which could be important hotspots for genome reconfiguration [30, 31]. In particular, the occurrence of large repeats in plastomes, such as the $307 \mathrm{bp}$ palindromic repeat observed in $P$. cuspidatus, has been speculated to result in an unstable genome structure due to inappropriate rearrangement [32]. In addition, these repeats provide many informative loci for the development of molecular markers for phylogenetics and population genetics [33]. As a very powerful type of molecular marker, SSRs are widely used in different research fields. They possess obvious advantages such as high polymorphism and cost effectiveness [34]. Most studies have shown that the predominant cpSSRs of land plants are consistent with their AT-biased plastomes. By contrast, the cpSSRs present in Polypodiaceae show considerable dissimilarity from the previously reported patterns. In this study, more than 38 SSRs were identified in every Polypodiaceae plastome, among which the majority were $\mathrm{C} / \mathrm{G}$ mononucleotides and were distributed in noncoding regions. The previously held idea that cpSSRs are generally composed of A/T repeats has now been challenged [35]. Gao et al. [36] have shown in denaturation experiments that repetitive structures with a higher GC content contribute to increasing the thermal stability of the Dryopteris fragrans plastome and maintaining its structure in the face of thermal changes. Species of Polypodiaceae evolved diversified morphological traits and lifestyles putatively in response to changes in terrestrial ecosystems caused by the radiation of angiosperms during the Cretaceous period [37]. Thus, we speculate that these repeating structures with a high GC content may be one of the molecular foundations of the adaptation of Polypodiaceae to the environment, which also provides new insights for understanding the environmental adaptation mechanism of plants. Furthermore, the cpSSRs developed in our study provide unique information for investigating genetic structure and genetic variation. In particular, these cpSSRs will be complementary and comparable to nuclear SSRs from ferns.

\section{Dynamic insertions in Polypodiaceae plastomes}

Although genome-wide alignment indicates that Polypodiaceae plastomes are rather conservative, we found abnormally large insertions in certain intergenic spacers. The large fragment insertion observed in the rrn16rps12 intergenic spacer of L. clathratus plastome is consistent with previous findings [16]. Similar insertion sequences have been detected in the LSC regions of some distantly related species and in the mitogenome of Asplenium nidus, implying that such sequences can move between genomic compartments [16]. Robison et al. [17] discovered a similar suite of dynamic mobile elements through an extensive investigation on fern plastomes, shedding light on the presence of MORFFO elements relative to inversions, intergenic expansions, and changes to inverted repeats. In this study, we characterized a completely different set of insertion sequences in the plastomes of Polypodiaceae. There are only two insertions that overlap with the morffo1 sequence, which are located in the petA-psaJ spacer in the LSC region of $P$. bonii and the rrn16-rps 12 spacer in the IR region of $P$. bifurcatum. The detected morffo 2 sequences are located in the $y c f 1-c c s \mathrm{~A}$ region at the IR/ LSC border in S. yakushimensis and the rrn16-rps12 spacer in the IR region of $P$. bifurcatum, showing no homology with other insertions. Our results further confirm the universality of MORFFO sequences in fern plastomes, and the presence of such a sequence at the inversion endpoint in S. yakushimensis suggests that the MORFFO sequences may be related to inversion events.

Although MORFFO sequences were not detected in the remaining Polypodiaceae species, there is another set of highly mobile insertions present in their plastomes. It is worth noting that plastid genes are rarely gained or lost, whereas our study indicates that the identified insertion sequences have been gained and lost frequently during the evolution of Polypodiaceae plastomes. This fluidity could indicate that these insertions in plastomes act as mobile elements. Furthermore, these insertions are frequently found adjacent to regions where more dispersed repeats occur. Many studies have shown that genomic rearrangement is related to small dispersed repeats (SDRs), which contribute to the repair mechanism induced by double-strand breaks [38, 39]. SDRs usually make an important contribution to the repetitive space in highly rearranged genomes and increase structural polymorphism even in closely related lineages, and they are mainly present in noncoding DNA fragments and related to small hairpin structures [38]. We speculate that 
the presence of rich repetitive motifs combined with highly mobile insertions may constitute the "trigger mechanism" for genome rearrangement in the plastomes of Polypodiaceae species, which can induce structural changes in the plastome under certain conditions. The limitations of the current sequence data for ferns mean that it is difficult to determine the exact source of these insertion sequences. As more genomic data are published in the future, the source and migration mechanisms of these insertion sequences should become clear.

\section{Conclusions}

As additional plastomes from Polypodiaceae are characterized, we obtain a clearer picture of plastome evolution for the family. It is generally considered that the evolution of Polypodiaceae plastomes is conservative, and their structural features are almost invariant in this family. Against this conservative background, however, the S. yakushimensis plastome stands out as unusual. The large-scale expansion of IRs and a unique inversion distinguish the S. yakushimensis plastome from those of all other Polypodiaceae studied thus far. In addition, many large mobile insertions are found in the plastomes of Polypodiaceae species, which often flank the dispersed repeated elements in the different Polypodiaceae plastomes. These unusual features are found in the structurally stable plastomes of Polypodiaceae and may therefore be implicated in the dynamic evolution of the plastomes of the family. In other words, unlike the static plastomes of Polypodiaceae characterized previously, the plastomes characterized herein are structurally unstable, as evidenced by the large mobile insertions found in Polypodiaceae.

\section{Methods}

\section{Sample collection, DNA extraction, and sequencing}

In this study, fresh leaves of $N$. fortunei, $N$. ovatus, and $P$. cuspidatus were sampled from the living collection from the South China Botanical Garden, Chinese Academy of Sciences (CAS), quickly frozen in liquid nitrogen, and stored at ultra-low- temperature refrigerator at $-80^{\circ} \mathrm{C}$ until use. Voucher specimens were deposited in the Herbarium of Sun Yat-sen University (SYS; voucher: S. Liu 201,630, S. Liu 201,654, and S. Liu 201,701 for N. fortunei, N. ovatus, and P. cuspidatus, respectively). Genomic DNA was extracted using the Tiangen Plant Genomic DNA Kit according to the manufacturer's instructions (Tiangen Biotech Co., Beijing, China). DNA quality was inspected in $0.8 \%$ agarose gels, and DNA quantification was performed using a NanoDrop spectrophotometer (Thermo Scientific, Carlsbad, CA, USA). After quality assessment, $500 \mathrm{ng}$ of DNA was sheared to an average fragment size of $300 \mathrm{bp}$ with a Covaris M220 ultrasonicator
(Covaris Inc., MS, USA). An Illumina paired-end (PE) sequencing library was constructed using the NEBNext, Ultra DNA Library Prep Kit (New England BioLabs Inc., Ipswich, MA). Sequencing took place on the HiSeq 2500 platform (Illumina Inc., San Diego, USA). Illumina sequencing produced approximately $2 \mathrm{~Gb}$ of raw data for each species.

\section{Genome assembly and annotation}

Raw reads were assessed for quality with FastQC v0.10.0 [40]. Low-quality bases $(\mathrm{Q}<20)$ and adapter sequences were trimmed by using Trimmomatic v0.32 [41]. Clean reads were mapped against the reference plastome of Lepisorus clathratus (NC_035739) to filtered chloroplast data. All mapped reads were de novo assembled into contigs with Velvet [42], and were further aligned and oriented with the reference genome. Remaining gaps were filled by direct PCR using specific primers that were designed based on contig sequences or homologous sequence alignments. Chloroplast gene annotation was conducted using DOGMA [43], followed by manual correction of the start and stop codons and intron/exon boundaries based on homologous genes from other published closely related fern plastomes. Transfer RNA (tRNA) genes were verified using ARAGORN [44] and tRNAscan-SE in organellar search mode with default parameters [45]. A circular map of the plastome was drawn with OGDRAW [46]. The accession numbers of $N$. fortunei, N. ovatus, and P. cuspidatus were MT373087, MT364352, and MT364353, respectively.

\section{Comparative analysis of Polypodiaceae plastomes}

The availability of multiple plastomes from Polypodiaceae provides an opportunity to explore the diversity of the genome within the family, including genome size and structure, GC content, gene order, and IR expansion/contraction. Therefore, we performed comparative analyses of three assembled plastome sequences and the nine other available plastomes of Polypodiaceae species, from Polypodiodes niponica (NC_040221), Lepisorus clathratus (NC_035739), Leptochilus hemionitideus (NC_040177), Lemmaphyllum microphyllum (MN623356), Platycerium bifurcatum (MN623367), Lepidomicrosorum hederaceum (MN623364), Pyrrosia bonii (NC_040226), Selliguea yakushimensis (MN623352), and Drynaria roosii (KY075853). The plastome of $D$. roosii was reannotated using gene prediction tools and manual adjustments before our analyses because of errors that we noticed in the $D$. roosii annotations. The genome size, gene content, IR boundaries, and base composition were compared based on the sequence and annotation information of these plastomes. Whole-genome alignments among the 12 Polypodiaceae species were performed to identify inversions using the ProgressiveMauve algorithm in Mauve 
v2.4.0 [47] after one inverted repeat (IR) copy was removed from each plastome.

The overall similarities of the 12 Polypodiaceae plastomes were plotted using the mVISTA [48] online program in Shuffle-LAGAN mode with the annotations of $N$. fortunei as a reference. To estimate nucleotide diversity $(\mathrm{Pi})$ and mutational hotspots among Polypodiaceae species, we performed pairwise alignments of 12 plastomes in MAFFT v7.310 software [49], adjusted manually with BioEdit software [50] if necessary. The nucleotide diversity values $(\mathrm{Pi})$ of the aligned sequences were calculated via sliding window analysis by using DnaSP v5.0 [51] with window lengths and step sizes of 600 and $200 \mathrm{bp}$, respectively.

\section{Characterization of SSRs and repeat sequences}

Further comparisons between Polypodiaceae species were performed with the repetitive elements found in their chloroplast sequences. Simple sequence repeats (SSRs) were detected using the Perl script MISA [52] (MIcroSAtellite; http://pgrc.ipk-gatersleben.de/misa/), with minimal iterations of ten repeat motifs for mononucleotides, five for dinucleotide repeats, and four for tri-, tetra-, pentaand hexa-nucleotides. Tandem repeats in eight Polypodiaceae species were recognized using Tandem Repeats Finder v4.09 [23], with matches, mismatches, and indels set at 2,7 , and 7 , respectively. The parameter settings were 90 for the minimum alignment score and 500 for the maximum period size. REPuter [53] (http://bibiserv.techfak. uni-bielefeld.de/reputer/) was used to visualize the location and size of the dispersed repeats (forward, reverse, complementary, and palindromic repeat sequences) with a minimal repeat size of $30 \mathrm{bp}$ and a hamming distance of 3 .

\section{Supplementary Information}

The online version contains supplementary material available at https:/doi. org/10.1186/s12870-020-02800-x.

Additional file 1: Table S1. Primers used for plastomes gap closing. Table S2. Distribution of SSRs in different plastome regions of Polypodiaceae. Table S3. Information on dispersed repeats among Polypodiaceae plastomes.

Additional file 2: Figure S1. Alignment of rps 16 exons/introns for the three plastomes that we sequenced. The intron is deleted only in $N$. fortunei.

Additional file 3: Figure S2. Nucleotide diversity (Pi) in the plastomes of 12 Polypodiaceae species.

Additional file 4: Figure S3. Identity of large insert fragments in rrn16rps12 among the plastomes of L. clathratus, P. bifurcatum, and D. roosii.

\section{Abbreviations}

cp: Chloroplast; IR: Inverted repeat; rRNA: Ribosomal RNA; SSC: Small single copy; tRNA: Transfer RNA; LSC: Large single copy; SSR: Simple sequence repeat; SDR: Small dispersed repeat; MORFFO: Mobile Open Reading Frames in Fern Organelles

Acknowledgements

Not applicable.

\section{Authors' contributions}

TW conceived and designed the experiments; SL and YS collected and contributed sample material; ZW performed the experiments; SL analyzed the data and wrote the manuscript; TW and YS reviewed the manuscript critically. All authors read and approved the final manuscript.

\section{Funding}

This work was supported by the National Natural Science Foundation of China [31670200, 31770587, 31872670, and 32071781], the Natural Science Foundation of Guangdong Province, China [2016A030313320 and

2017A030313122], Science and Technology Planning Project of Guangdong Province, China [2017A030303007], Project of Department of Science and Technology of Shenzhen City, Guangdong, China [JCYJ20160425165447211, JCYJ20170413155402977, JCYJ20170818155249053, and

JCYJ20190813172001780], and Science and Technology Planning Project of Guangzhou City, China [201804010389]. The funders had no role in the design of the study, analysis of the data nor in writing the manuscript.

\section{Availability of data and materials}

Annotated sequences of plastomes of N. fortunei, N. ovatus, and P. cuspidatus were submitted to GenBank (https://www.ncbi.nlm.nih.gov/genbank/) under MT373087, MT364352, and MT364353 accession number, respectively. All data generated or analyzed during this study are included in the article and its additional files.

Ethics approval and consent to participate

Not applicable.

\section{Consent for publication}

Not applicable.

\section{Competing interests}

The authors declare that they have no competing interests.

\section{Author details}

'School of Life Sciences, Sun Yat-sen University, Guangzhou, Guangdong, China. ${ }^{2}$ Research Institute of Sun Yat-sen University in Shenzhen, Shenzhen, Guangdong, China. ${ }^{3}$ College of Life Sciences, South China Agricultural University, Guangzhou, Guangdong, China.

Received: 30 April 2020 Accepted: 16 December 2020

Published online: 07 January 2021

\section{References}

1. Douglas SE. Plastid evolution: origins, diversity, trends. Curr Opin Genet Dev. 1998;8:655-61

2. Wolfe KH, Li WH, Sharp PM. Rates of nucleotide substitution vary greatly among plant mitochondrial, chloroplast, and nuclear DNAs. Proc Natl Acad Sci U S A. 1987;84:9054-8.

3. Drouin $G$, Daoud $H$, Xia J. Relative rates of synonymous substitutions in the mitochondrial, chloroplast and nuclear genomes of seed plants. Mol Phylogenet Evol. 2008:49:827-31.

4. Smith DR. Mutation rates in plastid genomes: they are lower than you might think. Genome Biol Evol. 2015;7:1227-34.

5. Palmer JD, Soltis DE, Chase MW. The plant tree of life: an overview and some points of view. Am J Bot. 2004;91:1437-45.

6. Lehtonen S. Towards resolving the complete fern tree of life. PLoS One. 2011;6:e24851

7. Chen ZD, Yang T, Lin L, Lu LM, Li HL, Sun M, et al. Tree of life for the genera of Chinese vascular plants. J Syst Evol. 2016;54:277-306.

8. Page CN. The diversity of ferns, an ecological perspective. In: Dyer AF, editor. The experimental biology of ferns. London: Academic; 1979. p. 9-56.

9. Mower JP, Vickrey TL. Structural diversity among plastid genomes of land plants. Adv Bot Res. 2018;85:263-92.

10. Wolf $P G$, Rowe $C A$, Sinclair RB, Hasebe M. Complete nucleotide sequence of the chloroplast genome from a leptosporangiate fern, Adiantum capillusveneris L. DNA Res. 2003;10:59-65.

11. Hasebe M, Iwatsuki K. Gene localization on the chloroplast DNA of the maiden hair fern; Adiantum capillus-veneris. Bot Mag Tokyo. 1992;105:413-9.

12. PPG I. A community-derived classification for extant lycophytes and ferns. J Syst Evol. 2016;54:563-603. 
13. Schuettpelz E, Pryer KM. Evidence for a Cenozoic radiation of ferns in an angiosperm-dominated canopy. Proc Natl Acad Sci U S A. 2009;106:11200-5.

14. Gentry AH, Dodson C. Diversity and biogeography of neotropical vascular epiphytes. Ann Mo Bot Gard. 1987;74:205-33.

15. Benzing DH. Vascular epiphytes. In: Lowman MD, Rinker BH, editors. Forest canopies. San Diego: Elsevier; 2004. p. 175-211.

16. Logacheva MD, Krinitsina AA, Belenikin MS, Khafizov K, Konorov EA, Kuptsov $S V$, et al. Comparative analysis of inverted repeats of polypod fern (Polypodiales) plastomes reveals two hypervariable regions. BMC Plant Biol. 2017;17:255.

17. Robison TA, Grusz AL, Wolf PG, Mower JP, Fauskee BD, Sosa K, et al. Mobile elements shape plastome evolution in ferns. Genome Biol Evol. 2018;10: 2558-71.

18. Shaw J, Lickey EB, Beck JT, Farmer SB, Liu W, Miller J, et al. The tortoise and the hare II: relative utility of 21 noncoding chloroplast DNA sequences for phylogenetic analysis. Am J Bot. 2005;92:142-66.

19. Shaw J, Lickey EB, Schilling EE, Small RL. Comparison of whole chloroplast genome sequences to choose noncoding regions for phylogenetic studies in angiosperms: the tortoise and the hare III. Am J Bot. 2007:94:275-88.

20. Kim KJ, Lee HL. Complete chloroplast genome sequences from Korean ginseng (Panax schinseng Nees) and comparative analysis of sequence evolution among 17 vascular plants. DNA Res. 2004;11:247-61.

21. Li YC, Korol AB, Fahima T, Beiles A, Nevo E. Microsatellites: genomic distribution, putative functions and mutational mechanisms: a review. Mol Ecol. 2002;11:2453-65.

22. Guichoux E, Lagache L, Wagner $S$, Chaumeil $P$, Léger $P$, Lepais $O$, et al. Current trends in microsatellite genotyping. Mol Ecol Resour. 2011;11: 591-611.

23. Benson G. Tandem repeats finder: a program to analyze DNA sequences. Nucleic Acids Res. 1999;27:573-80.

24. Burland TG. DNASTAR's Lasergene sequence analysis software. In: Misener S, Krawetz SA, editors. Bioinformatics methods and protocols. Totowa: Humana; 2000. p. 71-91.

25. Altschul SF, Gish W, Miller W, Myers EW, Lipman DJ. Basic local alignment search tool. J Mol Biol. 1990;215:403-10.

26. Madden T. The BLAST sequence analysis tool. 2nd ed: National Center for Biotechnology Information (US); 2013.

27. Yamada T. Repetitive sequence-mediated rearragements in Chlorella ellipsoidea chloroplast DNA: completion of nucleotide sequence of the large inverted repeat. Curr Genet. 1991;19:139-47.

28. Palmer JD. Comparative organization of chloroplast genomes. Annu Rev Genet. 1985:19:325-54

29. Khakhlova O, Bock R. Elimination of deleterious mutations in plastid genomes by gene conversion. Plant J. 2006;46:85-94.

30. Asano T, Tsudzuki T, Takahashi S, Shimada H, Kadowaki Kl. Complete nucleotide sequence of the sugarcane (Saccharum officinarum) chloroplast genome: a comparative analysis of four monocot chloroplast genomes. DNA Res. 2004;11:93-9.

31. Gao L, Yi X, Yang YX, Su YJ, Wang T. Complete chloroplast genome sequence of a tree fern Alsophila spinulosa: insights into evolutionary changes in fern chloroplast genomes. BMC Evol Biol. 2009:9:130.

32. Maréchal A, Brisson N. Recombination and the maintenance of plant organelle genome stability. New Phytol. 2010;186:299-317.

33. Chen C, Zheng Y, Liu S, Zhong Y, Wu Y, Li J, et al. The complete chloroplast genome of Cinnamomum camphora and its comparison with related Lauraceae species. PeerJ. 2017;5:e3820.

34. Wang ML, Barkley NA, Jenkins TM. Microsatellite markers in plants and insects. Part I Appl Biotechnol G3. 2009;3:54-67.

35. Kuang DY, Wu H, Wang YL, Gao LM, Zhang SZ, Lu L. Complete chloroplast genome sequence of Magnolia kwangsiensis (Magnoliaceae): implication for DNA barcoding and population genetics. Genome. 2011;54:663-73.

36. Gao R, Wang W, Huang Q, Fan R, Wang X, Feng P, et al. Complete chloroplast genome sequence of Dryopteris fragrans (L.) Schott and the repeat structures against the thermal environment. Sci Rep. 2018;8:1-11.

37. Schneider $H$, Schuettpelz E, Pryer KM, Cranfill R, Magallón S, Lupia R. Ferns diversified in the shadow of angiosperms. Nature. 2004;428:553-7.

38. Milligan BG, Hampton JN, Palmer JD. Dispersed repeats and structural reorganization in subclover chloroplast DNA. Mol Biol Evol. 1989;6:355-68.

39. Odom OW, Baek KH, Dani RN, Herrin DL. Chlamydomonas chloroplasts can use short dispersed repeats and multiple pathways to repair a doublestrand break in the genome. Plant J. 2008:53:842-53.
40. Andrews S. FastQC a quality control tool for high throughput sequence data. http://www.bioinformatics.babraham.ac.uk/projects/fastqc/.

41. Bolger AM, Lohse M, Usadel B. Trimmomatic: a flexible trimmer for Illumina sequence data. Bioinformatics. 2014;30:2114-20.

42. Zerbino DR, Birney E. Velvet: algorithms for de novo short read assembly using de Bruijn graphs. Genome Res. 2008;18:821-9.

43. Wyman SK, Jansen RK, Boore JL. Automatic annotation of organellar genomes with DOGMA. Bioinformatics. 2004;20:3252-5.

44. Laslett D, Canback B. ARAGORN, a program to detect tRNA genes and tmRNA genes in nucleotide sequences. Nucleic Acids Res. 2004;32:11-6.

45. Schattner P, Brooks AN, Lowe TM. The tRNAscan-SE, snoscan and snoGPS web servers for the detection of tRNAs and snoRNAs. Nucleic Acids Res. 2005;33:W686-W9.

46. Lohse M, Drechsel O, Kahlau S, Bock R. OrganellarGenomeDRAW—a suite of tools for generating physical maps of plastid and mitochondrial genomes and visualizing expression data sets. Nucleic Acids Res. 2013;41:W575-W81.

47. Darling AC, Mau B, Blattner FR, Perna NT. Mauve: multiple alignment of conserved genomic sequence with rearrangements. Genome Res. 2004;14: 1394-403.

48. Frazer KA, Pachter L, Poliakov A, Rubin EM, Dubchak I. VISTA: computational tools for comparative genomics. Nucleic Acids Res. 2004;32:W273-W79.

49. Katoh K, Standley DM. MAFFT multiple sequence alignment software version 7: improvements in performance and usability. Mol Biol Evol. 2013 30:772-80.

50. Hall TA. BioEdit: a user-friendly biological sequence alignment editor and analysis program for windows 95/98/NT. Nucleic Acids Symp Ser. 1999;41: 95-8.

51. Librado P, Rozas J. DnaSP v5: a software for comprehensive analysis of DNA polymorphism data. Bioinformatics. 2009;25:1451-2.

52. Beier S, Thiel T, Münch T, Scholz U, Mascher M. MISA-web: a web server for microsatellite prediction. Bioinformatics. 2017;33:2583-5.

53. Kurtz S, Choudhuri JV, Ohlebusch E, Schleiermacher C, Stoye J, Giegerich R. REPuter: the manifold applications of repeat analysis on a genomic scale. Nucleic Acids Res. 2001;29:4633-42

\section{Publisher's Note}

Springer Nature remains neutral with regard to jurisdictional claims in published maps and institutional affiliations.

\section{Ready to submit your research? Choose BMC and benefit from:}

- fast, convenient online submission

- thorough peer review by experienced researchers in your field

- rapid publication on acceptance

- support for research data, including large and complex data types

- gold Open Access which fosters wider collaboration and increased citations

- maximum visibility for your research: over $100 \mathrm{M}$ website views per year

At $\mathrm{BMC}$, research is always in progress.

Learn more biomedcentral.com/submissions 